\title{
Temporal evolution of neoclassical tearing modes in the frequently interrupted regime
}

\author{
O. Dumbrajs ${ }^{1}$, V. Igochine ${ }^{2}$, A. Gude ${ }^{2}$, M. Maraschek ${ }^{2}$, H. Zohm ${ }^{2}$ and ASDEX Upgrade Team ${ }^{2}$ \\ ${ }^{1}$ Institute of Solid State Physics, Association Euratom-University of Latvia, Kengaraga Street 8, LV-1063, Riga, Latvia \\ ${ }^{2}$ MPI für Plasmaphysik, Euratom-Association, D-85748 Garching, Germany
}

\begin{abstract}
A phenomenological method for description of temporal evolution of neoclassical tearing modes in the frequently interrupted regime (FIR) is proposed. The method makes it possible to predict the beginning and the end of the FIR regime as well as the frequency of the FIR drops. A few experimental parameters which are used in the model are commonly measured quantities. Several specific ASDEX Upgrade (http://en.wikipedia.org/wiki/ASDEX_Upgrade) FIR discharges with different heating and different FIR behaviour are analyzed in detail.
\end{abstract}

PACS numbers: 52.35.Vd, 52.35.Py, 52.25.Xz, 52.55.Fa, 52.55.Tn, 02.50.Ey

\section{INTRODUCTION}

An important goal of fusion research with magnetically confined plasmas is to maximize the achievable fusion performance. In a tokamak, neoclassical tearing modes (NTMs), i.e. magnetic islands driven unstable by the loss of bootstrap current inside the island are of major concern, as they are considered to be the most severe limitation to the maximum achievable plasma pressure in conventional tokamak scenarios. Such instabilities involve considerably large displacements of the plasma. On ASDEX Upgrade a regime has been found $[1,2]$ when the amplitude of the NTM after reaching a certain size suddenly drops to a much smaller value. After this the mode growth starts again. In this way the NTM amplitude never reaches its stationary saturated value. This kind of neoclassical tearing modes was called frequently interrupted regime (FIR)-NTMs. In particular it has been observed that the amplitude of the $(m, n)=(3,2)$ NTM ( $m$ and $n$ are the poloidal and 
toroidal mode number, respectively) drops as an additional MHD instability, the $(m, n)=(4,3)$ mode, occurs. Generally the occurrence of the $(m+1, n+1)$ modes always coincides with the $(1,1)$ mode activity which is a necessary condition for the nonlinear coupling to the $(m, n)$ NTM. The time in which these amplitude drops occur is very short (about $500^{\mu s}$ ), much shorter than the resistive MHD reconnection rate (few 10 s of milliseconds in the ASDEX Upgrade). It has been suggested $[2,3]$ that this experimental observation can be explained by stochastization of magnetic field lines when the island separatrix is destroyed. In $[4,5]$ the mapping technique was used to trace the magnetic field lines and evidence in favor of the stochastization hypothesis was found.

It should be noted that the mapping technique by itself is not able do describe temporal development of NTMs. In this paper we demonstrate how one can follow the temporal evolution of neoclassical tearing modes in the frequently interrupted regime.

\section{EQUATIONS}

We write the standard Rutherford equation modified by the bootstrap current effects in the following form

$$
\frac{\partial W}{\partial t}=\frac{r_{r e s}}{\tau_{r}}\left[r_{r e s} \Delta^{\prime}-\varepsilon^{1 / 2} \frac{1}{S W} \cdot \frac{2 \nabla p r_{r e s}^{2}}{B_{p}^{2}}\right]
$$

Here $W$ is the $(3,2)$ island width, $\tau_{r}=4 \pi r L^{2} /\left(\eta c^{2}\right)(L \approx 5 \mathrm{~cm} \cdot 1.5)$ is the resistive timescale for evolution of NTM, $\quad \eta=m_{e} v_{T e} /\left(e^{2} n_{e}\right)$ is plasma resistivity, $v_{T e}=2.91 \times 10^{-6} n_{e} \ln \Lambda T e^{-3 / 2}$ is electron collision rate, $\varepsilon=a / R$ is the inverse aspect ratio, $a$ and $R$ are minor and major radii of the torus, respectively, $q$ is the safety factor at the rational surface, $r_{\text {res }}$ is the radius of the surface, $\Delta^{\prime}=-m / r_{\text {res }}$ is the usual tearing stability parameter, $S=r_{\text {res }} \nabla q / q$ is shear, $\nabla p$ is the plasma pressure gradient at the resonant surface, and $B_{p}$ is the poloidal magnetic field. This equation does not include further corrections due to small island size (polarization currents, driven toroidal mode coupling, 
rotation physics, etc). There are two reasons for this: (i) there is no final theory which could answer on the value of such terms, (ii) in this paper we consider only sufficiently large magnetic islands and the small island terms which are responsible for initial growth of the island are not important in our case (see $[6,7,8]$ and references therein which deal with the generalized Rutherford equation and discuss different factors related to seeding island terms).

Equation (1) would describe correctly the temporal evolution of $W$ (not the FIR phenomenon), if the functions $\nabla p(t)$ and $S(t)$ were known. To account for the FIR phenomenon, one has to describe the onset of the ideal $(m+1, n+1)$ mode which also depends on $\nabla p(t)$ and $S(t)$ [3]. It was shown [9] that in the presence of an island close to the ideal kink resonant surface the stability properties of the ideal kink mode are strongly changed and an initially stable ideal mode could be destabilized. However, $\nabla p(t)$ and $S(t)$ are hardly extractable from measurements. Even in the case of an accurately measured safety factor and pressure profiles, the errors of their gradients remain large. It is well known that increasing normalized pressure, $\beta_{N}$ leads to an increase of the pressure gradient. $\left(\beta_{N}=\beta_{t} a B_{t} / I_{p l}, \beta_{t}=2 \mu_{0}\langle p\rangle / B_{t}\right), B_{t}$ : toroidal magnetic field, $\langle p\rangle$ : averaged plasma pressure, $I_{p l}$ : plasma current.) We assume that the knowledge of $\nabla p(t)$ and $S(t)$ can be replaced by the experimental information about the normalized pressure: $-\nabla p / S=\alpha \beta_{N}$. The exact theoretical justification of such an assumption does not exist but it works very well as it is shown in what follows. The influence of the $(3,2)$ island on the stability of the $(4,3)$ mode is included through the island width. The critical $(3,2)$ island width $W_{\text {crit }}$, at which the ideal $(4,3)$ island becomes unstable and FIR-drops occur, can be routinely measured in experiments. Thus, in our case, growth of the $(3,2)$ mode destabilizes the $(4,3)$ ideal mode as soon as $W=W_{c r i t}$, which is only possible once $\beta_{N}$ is above a critical value. Essentially this means that the border line of stability of the $(4,3)$ ideal kink mode can be traced in the $\left(W, \beta_{N}\right)$ plane. With these assumptions Eq. (1) can be rewritten as follows: 


$$
\frac{\partial W}{\partial t}=\frac{r_{r e s}}{\tau_{r}}\left[r_{r e s} \Delta^{\prime}+\varepsilon^{1 / 2} \frac{1}{W} \cdot \frac{2 r_{r e s}^{2}}{B_{p}^{2}} \alpha \beta_{N}\right] \quad \text { for } W<W_{c r i t}
$$

where $\alpha$ is a free parameter which has to be determined from the experiment. As it will be shown in what follows, this assumption gives good predictions for periodicity of FIRdrops for standard discharges without special local changes of the shear around the resonant surface, e.g. by local current drive.

Equation (2) describes the temporal evolution of $W$ until it reaches the critical value $W_{\text {crit }}$. At this instance the $(3,2)$ mode begins to decay with the rate $\gamma$

$$
W=W_{c r i t} \exp (-\varkappa)
$$

which is assumed to be equal to the growth rate of the $(4,3)$ mode. The further temporal evolution of $W$ during the FIR drop is described by the equation

$$
\frac{\partial W}{\partial t}=\frac{r_{r e s}}{\tau_{r}}\left[r_{r e s} \Delta^{\prime}+\varepsilon^{1 / 2} \frac{1}{W} \cdot \frac{2 r_{r e s}^{2}}{B_{p}^{2}} \alpha \beta_{N}\right]-W \gamma
$$

until $W_{\text {slides down to }} W_{\text {min }}$, the $(4,3)$ mode vanishes and the FIR crash is completed. After that $W$ begins to grow again and the cycle repeats. The decay term $W \gamma$ is dominant during the FIR drop. The amplitude of the $(3,2)$ island from equation (2) is almost constant during this phase while the amplitude of the ideal $(4,3)$ mode grows very fast as found in the experiment (for example see Figure 8 in Ref. 2). Assuming a stochastic mechanism, the FIR drop could be described as follows: The size of the stochastic area depends on the overlap of the $(3,2)$ and $(4,3)$ modes. Stochastization starts at the island separatrix and spreads out to both directions (inside the $(3,2)$ island and towards the $(4,3)$ surface) with growing $(4,3)$ amplitude. The $(4,3)$ flux surface itself remains unchanged until the stochastic area gets very close to it. Thereby the driving force for the $(4,3)$ kink mode is maintained until a certain $(4,3)$ size it reached, although the perturbation current inside the $(3,2)$ island has already decreased. Here the stochastic area partially destroys 
the outer part of the $(3,2)$ island and thus reduces its size. It should be mentioned that any fast reduction of the island width would lead to similar predictions. Since only the stochastization hypothesis is able to explain the FIR regime presently, we prefer to use a "physically relevant" description instead of simple infinitely fast drop.

The values of $W_{\min }$ and $W_{\text {crit }}$ have been extracted from the measured ECE isotherms [3]. Alternatively ratios of $W_{\text {crit }}$ to $W_{\min }$ can also be found from Poincare plots produced by the mapping technique and attributed to stochastization during FIR as discussed in [4,5]. It is also possible to use Soft X-ray cameras and magnetic measurements for determination of $W_{c r i t}$ and $W_{\min }$.

In summary, the four parameters of the model which have to be determined from experiments are: i) $\alpha$ a fitting parameter related to the experimental $\beta_{N}(t)$ trace, ii) $W_{\text {crit }}$, iii) $W_{\text {crit }} / W_{\min }$, and iv) $\gamma$. The values of these parameters are easily extractable by means of standard diagnostics (magnetic, SXR, or ECE). In Sec. III we investigate the sensitivity of our model with respect to these parameters.

\section{EXAMPLES}

In the calculations the following numerical values of various ASDEX Upgrade parameters were used: $a=50 \mathrm{~cm}, R=165 \mathrm{~cm}, \quad r_{r e s}=29.6 \mathrm{~cm}, \quad T_{e}=2550 \mathrm{eV}$, $n_{e}=5 \cdot 10^{13} \mathrm{~cm}^{-3}, B_{p}=3000$ gauss $, v_{T e}=1.86 \cdot 10^{4} \mathrm{~s}^{-1}, \eta=1.47 \cdot 10^{-18} \mathrm{~s}, \tau_{R}=0.53 \mathrm{~s}$, $\Delta^{\prime}=-0.101 \mathrm{~cm}^{-1}$, and $\ln \Lambda=16.5$.

\section{A. Standard evolution of the FIR-NTM}

During the shown time range for the discharge 11681 (Fig. 1) the applied NBI heating power was $P_{N B I} \approx 10 M W$, the toroidal magnetic field was $B_{t}=2.2 T, q_{95}=4.6$ and $I_{p l}=0.8 M A$. Here $W_{c r i t} / W_{\min } \approx 1.25$ (see Table 1 in [2]). 
In solving Eq. (2) the parameter $\alpha=85500$ was chosen such that FIR continues until $t \approx 3.35 s$ which is known from experiment. Here as soon as $W$ reached $W_{\text {crit }}=7.0 \mathrm{~cm}$, also known from experiment (Table 1 in Ref. 2), Eq. (2) was replaced by Eq. (4) which was solved until $W=W_{\min }$. At this moment Eq. (2) was substituted again for Eq. (4), etc. Here $\gamma=1320 \mathrm{~s}^{-1}$ was used, as estimated on the basis of the $(4,3)$ burst time of $1 m s$ (see Fig. 8 in Ref. 2).

The results of the calculations are presented in Fig. 1. At the mode onset $\beta_{N}$ is well above the critical value, therefore the $(3,2)$ mode has FIR character. It can be seen that the period of the FIR crashes strongly depends on $\beta_{N}$. Increase of the normalized pressure leads to more frequent FIR crashes, decrease of the pressure results in longer times between the crashes. Finally, for low values of $\beta_{N}(t \geq 3.25 s)$ the island width no longer changes abruptly. It is worthy of notice that experimental and theoretical FIR periods $\tau_{F I R}$ are in good agreement (lower part of Fig. 1). $\tau_{F I R}$ is defined as time between two subsequent FIR drops which allows evaluation only from the second FIR drop time point. 

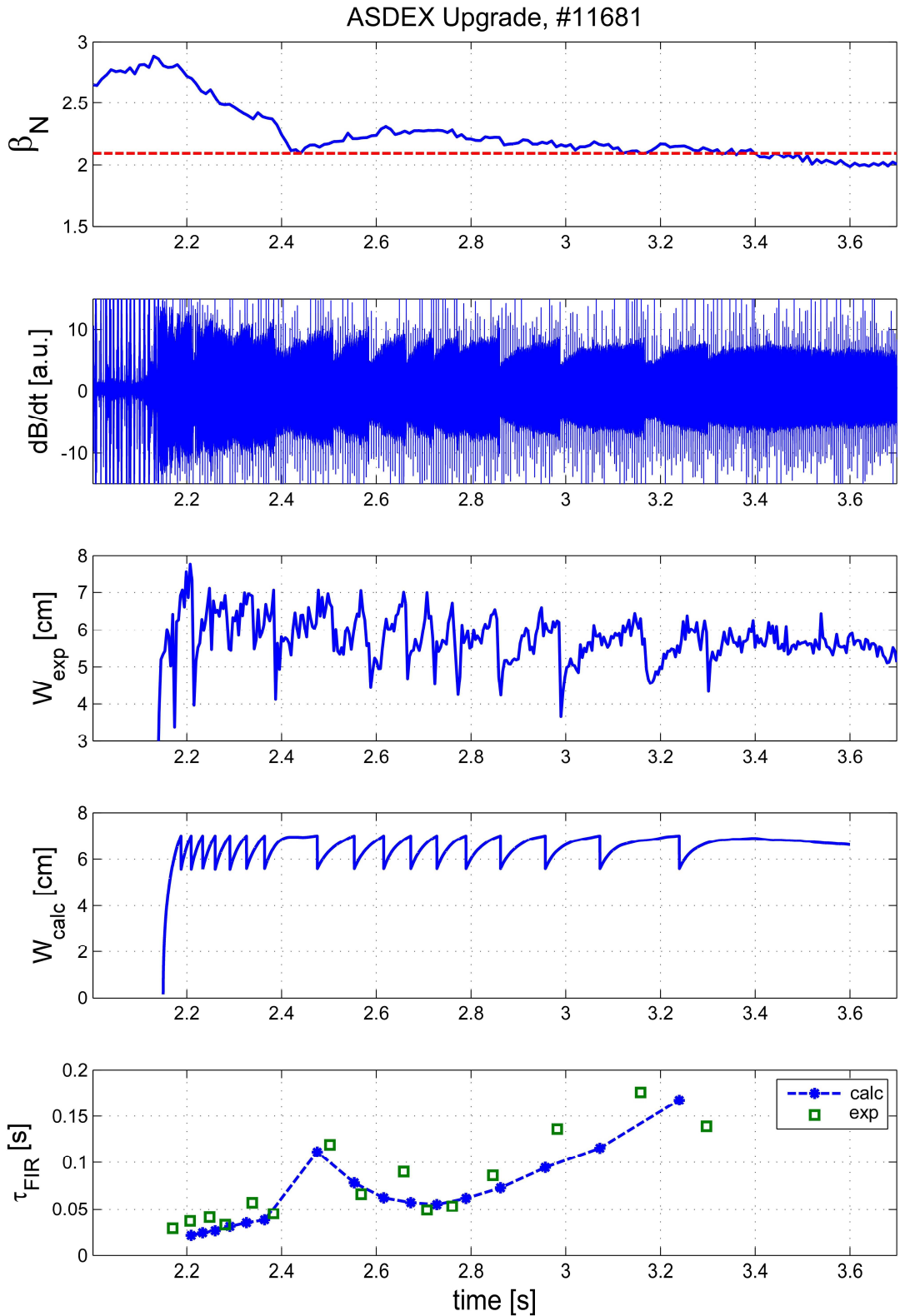

Figure 1. (Color online) Temporal evolution of $\beta_{N}, d B / d t, W_{\text {exp }}, W_{\text {calc }}$, and $\tau_{F I R}$. The red dashed line marks the border line between absence (below) and presence (above) of FIR phenomenon. The heating power was reduced by $2.5 \mathrm{MW}$ between $2.38 \mathrm{~s}$ and $2.42 \mathrm{~s}$ which causes the fast $\beta_{N}$ drop during this time. Here $\alpha=85500$. 

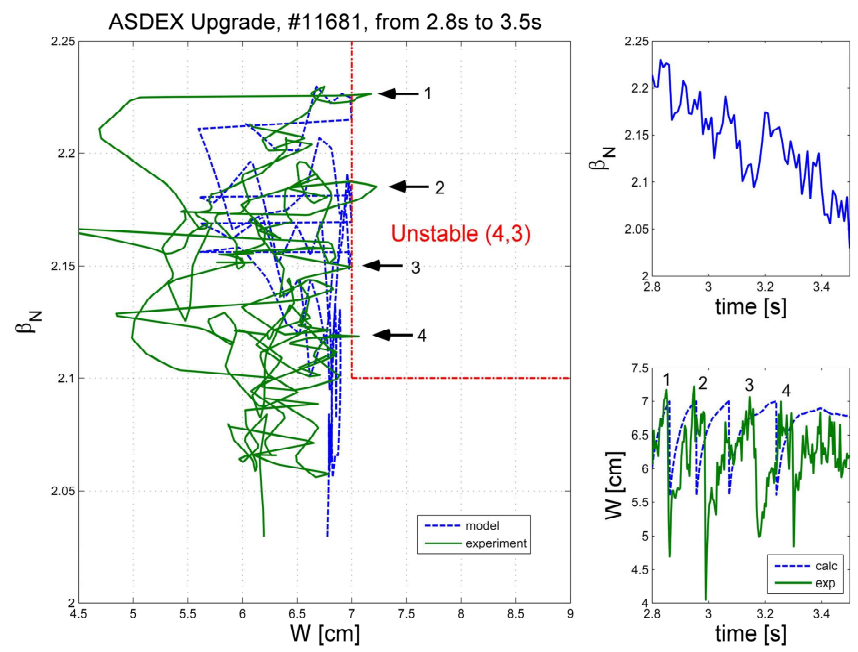

Figure 2. (Color online) FIR behaviour in the $\left(W, \beta_{N}\right)$ plane is shown for four FIR drops for the same discharge as in figure 1 . The ideal $(4,3)$ mode becomes unstable for $\mathrm{W}>\mathrm{W}_{\text {crit }}$ which causes the FIR drops in the $(3,2)$ amplitude. The experimental FIR drops are marked from 1 to 4 on the $\left(W, \beta_{N}\right)$ plane and on the time evolution plot.

The behaviour of a FIR-NTM in the $\left(W, \beta_{N}\right)$ space can be clearly seen both in modelled and in the experimental curves (figure 2). The ideal $(4,3)$ mode becomes unstable for $\mathrm{W}>\mathrm{W}_{\text {crit }}$ which causes the FIR drops in the $(3,2)$ amplitude. This verifies that, indeed, the border line of stability of the $(4,3)$ mode can be traced as a border line in the $\left(W_{c r i t}, \beta_{N}\right)$ plane. It is clear that the model for the drop phase is approximate and assumes for example infinitely fast mode coupling and other limitations, but all these limitations do not affect the long term behaviours which is investigated here. Finally it should be recalled that according to $[4,5]$ entry into FIR domain is related to stochastization.

\section{B. Increase of the heating during the NTM evolution and high $\beta_{N}$ case}

Variations of the applied heating are typical for many plasma discharges. Thus, it is necessary to test robustness of our approach also for cases with changes of the applied heating power during the NTM evolution. Two different examples are considered in what follows. The first case demonstrates increase of the heating power (\#11816, figure 3). 
Here $I_{p}=1.0 \mathrm{MA}$ and $B_{t}=2.56 T$. The NBI power was increased at $3.8 s$ and $4.4 \mathrm{~s}$ which leads to increase of $\beta_{N}$ and transition to the FIR regime. One can see a good agreement between experimental and modelled evolution of the NTM.
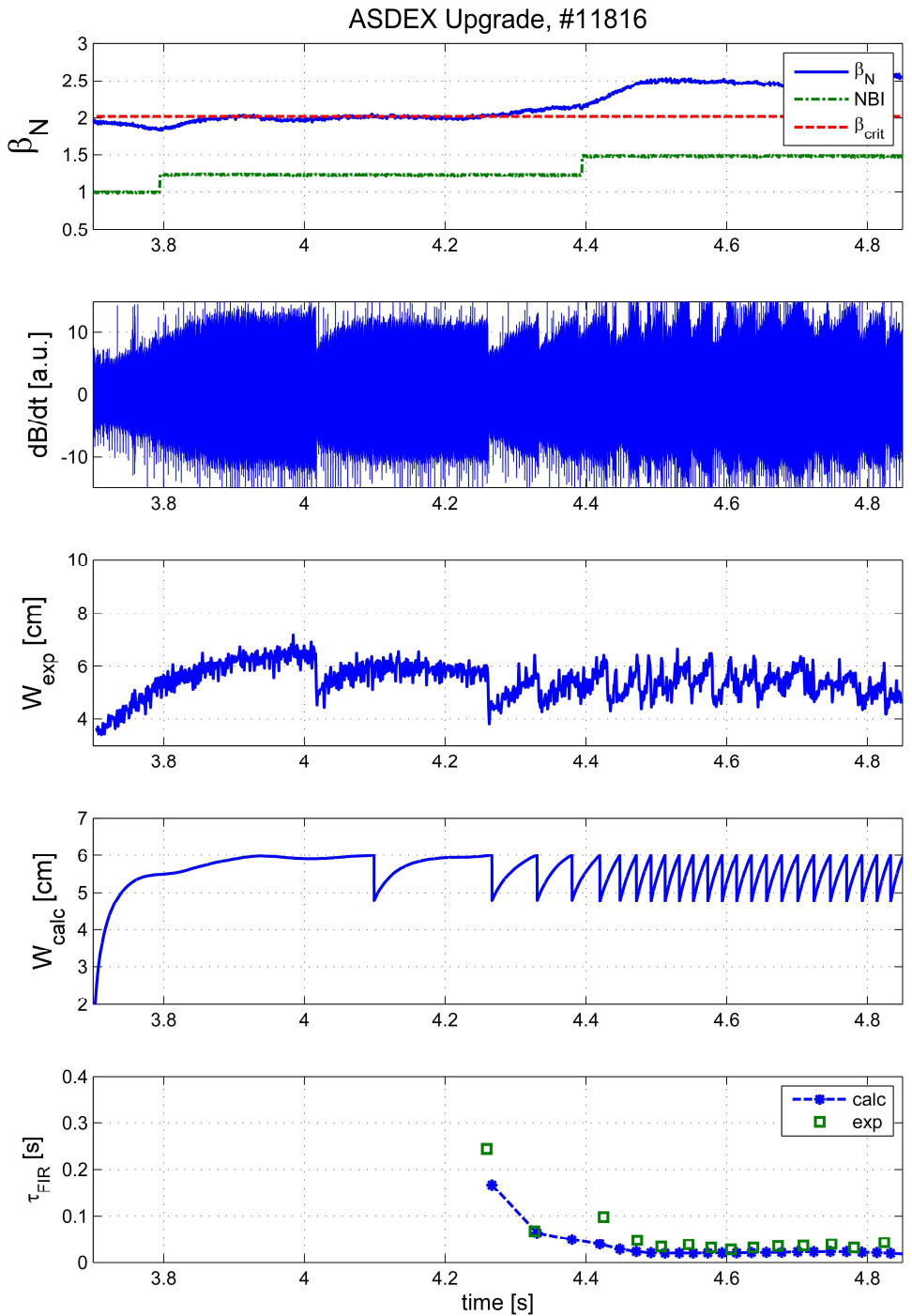

Figure 3. (Color online) Temporal evolution of $\beta_{N}, N B I, d B / d t, W_{\exp }, W_{\text {calc }}$, and $\tau_{F I R}$. The red dashed line marks the border line between absence (below) and presence (above) of FIR phenomenon. In this case $\beta_{N}$ is close to the critical value during the first phase which leads to very long time between first FIR drops. Increase of the heating power leads to more frequent FIR drops. Here $\alpha=78000$. 

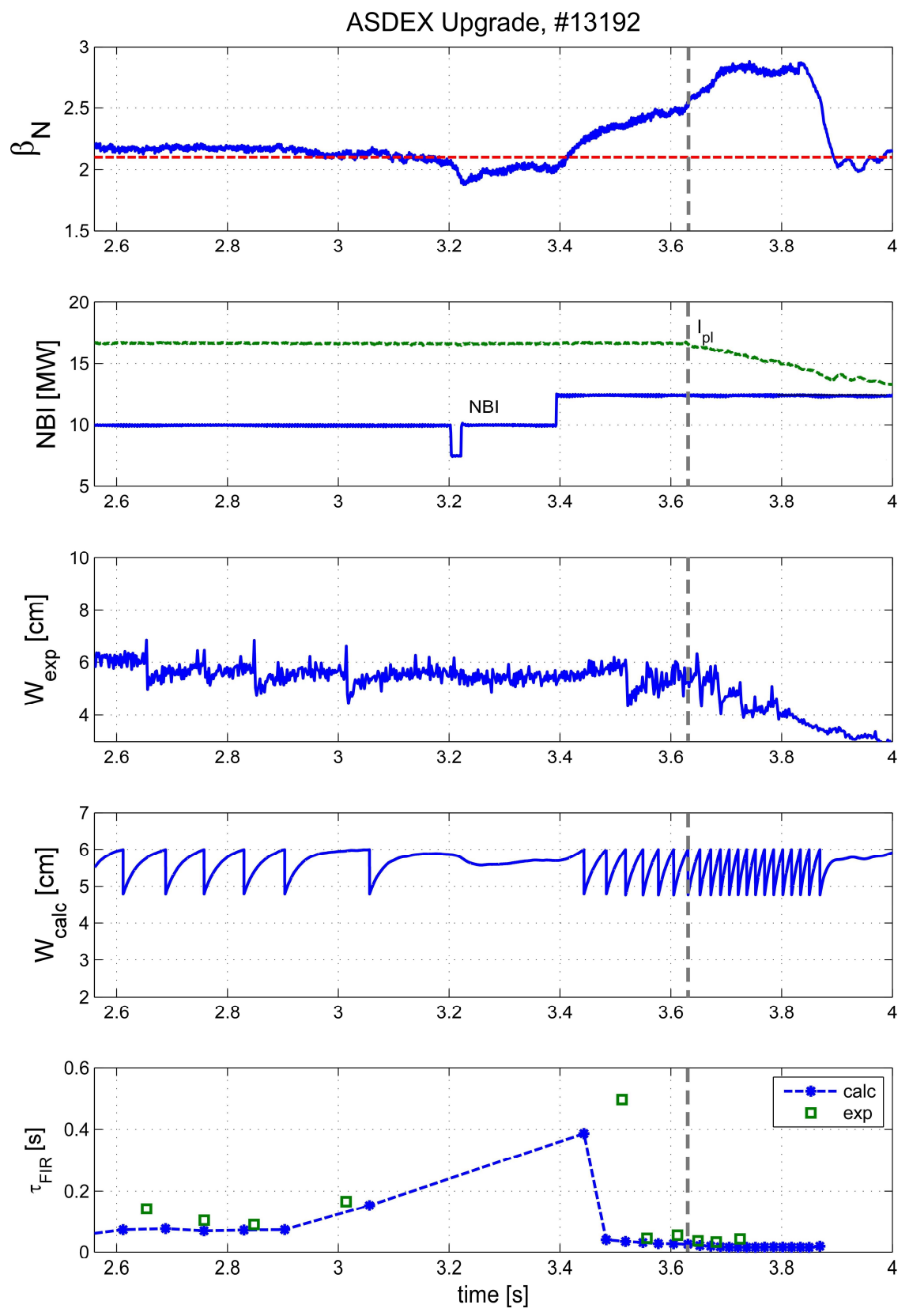

Figure 4. (Color online) Temporal evolution of $\beta_{N}, N B I, I_{p l}, W_{\text {exp }}, W_{c a l c}$, and $\tau_{F I R}$. The red dashed line marks the border line between absence (below) and presence (above) of FIR phenomenon. One can see good agreement between experimental and modelled evolution of the NTM. The FIR periods are also well 
reproduced up to the beginning of the current decay (at about $\mathrm{t}=3.61 \mathrm{~s}$, marked by a vertical line). Here $\alpha=74000$.

The second case shows a discharge with different plasma current and magnetic field $\left(I_{p}=0.8 M A, B_{t}=2.1 T\right)$. Experimental temporal evolution of $\beta_{N}, \mathrm{NBI}$, and $W_{\exp }$ for \#13192 discharge is shown in Fig. 4. This example is more challenging because an additional heating source was applied during a $\mathrm{B}_{\mathrm{t}}$ ramp and two transitions (FIR-NTM $\rightarrow$ NTM $\rightarrow$ FIR-NTM) should be traced. With the initially applied heating power $\left(P_{N B I}=10 M W\right) \beta_{N}$ was just above the critical value which resulted in weak FIR behaviour with long time between FIR drops. At $t=3.0-3.1 \mathrm{~s} \beta_{N}$ fell below the critical line and the FIR stopped. Subsequently the power was increased up to $12.5 \mathrm{MW}(\mathrm{t}=3.4 \mathrm{~s})$ which led to a strong increase of $\beta_{N}$ and the reappearance of FIR with short period. Results of the model with $\alpha=74000$ (lower part of Fig. 4) are in good qualitative agreement with these experimental observations in spite of the small $B_{t}$ ramp from $2.08 \mathrm{~T}$ to $2.16 \mathrm{~T}$ during this phase and $300 \mathrm{~kW}$ of electron cyclotron resonance heating (ECRH). This example shows that the present technique is sufficiently robust to be used for prediction and tracing of the FIR regime in most experimental cases in spite of its simplicity and restricted set of parameters. It is interesting that $\alpha$ is almost the same in these two examples $(\alpha=78000$ in $\# 11816 ; \alpha=74000$ in \#13192) in spite of different plasma currents and magnetic fields. The influence of different parameters in comparison with experimental values for the FIR period is shown in Fig. 5. One can see that 5\% variation of $\alpha$ does not change the result much. Variation of $\gamma$ has almost zero effect. This reflects the fact that the FIR drop is extremely fast in comparison with the FIR period. Variation of $W_{c r i t} / W_{\min }$ changes the results only slightly. All these variations are not larger than natural variations of the FIR period as observed in the experiments. 


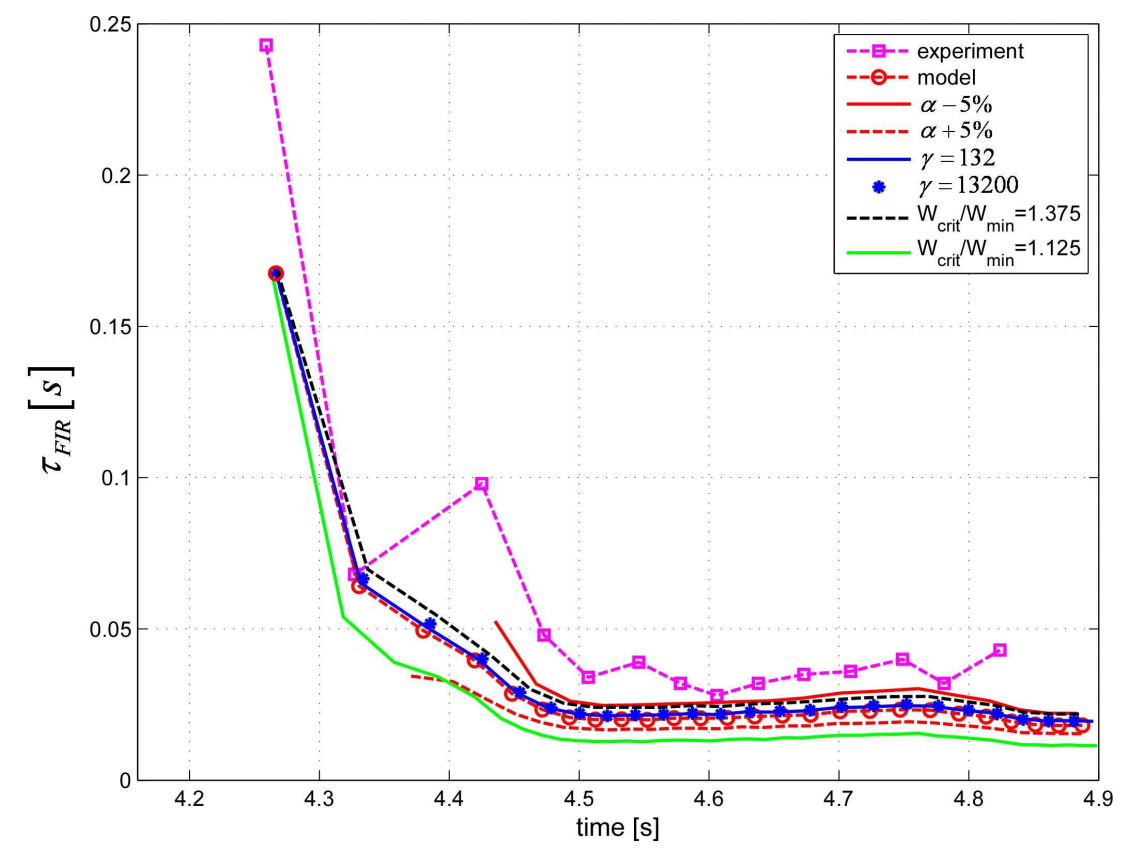

Figure 5. (Color online) Influence of different parameters on the FIR period in the case of discharge \#11816 (figure 3). Variation of $\alpha$ by $\pm 5 \%$ only slightly changes the result. Variation of $\gamma$ has almost zero effect, because in all cases the time growth rate is much faster than the FIR period. Variation of $W_{c r i t} / W_{\min }$ changes the results slightly.

It should be noted that local modification of shear at the resonant surface (for example with ECCD deposition) could lead to triggering of the FIR regime well below $\beta_{\text {crit }}$. In such cases our method cannot be directly applied and the analysis of the mode stability has to be done. This would require a detailed reconstruction of the plasma profiles and careful stability calculations. Such analysis is beyond the scope of the present paper.

\section{CONCLUSIONS}

A phenomenological method for description of temporal evolution of neoclassical tearing modes in the frequently interrupted regime (FIR) is proposed. The method implies modification of the Rutherford equation for time evolution of the NTM and uses well 
measured experimental quantities. The method makes it possible to predict the beginning and the end of the FIR regime as well as the frequency of the FIR drops for online control. A few experimental parameters which are used in the model are commonly measured quantities. This allows one to utilize the model during the experiment. Three different ASDEX Upgrade FIR discharges, representing a large range of discharges, have been analyzed in detail. Good agreement between the experimental and theoretical temporal evolution of the $(3,2)$ island size has been demonstrated with a very narrow range of free parameters: $\alpha$ values varied by only $\pm 7 \%, W_{\text {crit }}$ was between 6 and $7 \mathrm{~cm}$, and $W_{\text {crit }} / W_{\min }=1.25$ in all cases. Good agreement was also achieved for discharges with modification of the plasma heating during the NTM evolution. This suggests that the free parameters for this model, that in principle have to be determined from the experiment, do not vary much, neither during nor between different discharges. Together with the fact that the result is not much affected by variations of input parameters, the present technique seems to be sufficiently robust for prediction and tracing of the FIR regime for a large range of discharges. This also suggests that for other discharge types, where the parameter values found here are not valid, it should be possible to determine the values from some discharges and then use them for prediction. It is clear that the model does not predict the exact time of the FIR crashes but it gives good estimation for the beginning and the end of the FIR regime as well as for the FIR frequency. These results are sufficient for discharge performance control. The exact time of the crashes is not important for the online control. 


\section{References}

[1] Günter S., Gude A., Maraschek M., Sesnic S., Zohm H., and the ASDEX Upgrade Team, and Howell D.F. 2001 Phys. Rev. Lett. 87, 275001.

[2] Gude A., Günter S., Maraschek M., Zohm H., and the ASDEX Upgrade Team, 2002 Nucl. Fusion 42, 833.

[3] Günter S., Maraschek M., de Baar M., Howell D.F., Poli E., Strumberger E., Tichmann C., ASDEX Upgrade Team, and Contributors to the EFDA-JET Workprogramme, 2004 Nucl. Fusion 44, 524.

[4] Dumbrajs O., Igochine V., Constantinescu D., and Zohm H. 2005 Phys. Plasmas 12 110704

[5] Igochine V., Dumbrajs O., Constantinescu D., Zohm H., and Zvejnieks G. 2006 Nucl. Fusion 46, 741

[6] O. Sauter, R. J. La Haye, Z. Chang, D. A. Gates, Y. Kamada, H. Zohm, A. Bondeson, D. Boucher, J. D. Callen, M. S. Chu, T. A. Gianakon, O. Gruber, R. W. Harvey, C. C. Hegna, L. L. Lao, D. A. Monticello, F. Perkins, A. Pletzer, A. H. Reiman, M. Rosenbluth, E. J. Strait, T. S. Taylor, A. D. Turnbull, F. Waelbroeck, J. C. Wesley, H. R. Wilson and R. Yoshinof, 1997 Phys. Plasmas 4, 1654

[7] R.J. Buttery, T.C. Hender, D.F. Howell, R.J. La Haye, S. Parris, O. Sauter, C.G. Windsor and JET-EFDA Contributors, 2004 Nuclear Fusion 44, 678

[8] R.J. La Haye, R. Prater, R.J. Buttery, N. Hayashi, A. Isayama, M. Maraschek, L. Urso and H. Zohm, Nucl. Fusion 46 (2006) 451-461

[9] Hegna C.C. and Callen J.D., 1992 Phys. Fluids B 4, 3031 